\title{
Comportamiento "in vivo" de aislamientos de Trichomonas vaginalis, utilizando ratones NMRI como modelo experimental
}

\author{
LÁZARA ROJAS R., IDALIA SARIEGO R., CARLOS SARRÍA P. y JORGE FRAGA N.
}

\section{Behavior "in vitro" of isolations of Trichomonas vaginalis, using mice NMRI as experimental model}

The correlation between clinical severity of vaginal trichomoniasis in adolescents and Trichomonas vaginalis virulence in an experimental model was studied. For this purpose, four hundred mice were inoculated intraperitoneally with 40 Trichomonas vaginalis isolates obtained from vaginal exudates. These were obtained from adolescents clinically classified as asymptomatic or mild, moderate or severe symptomatic patients, attending the Infant-Juvenile Service of Pregnancy Interruption and Sexually-Transmitted Diseases from Gynecoobstetrics Hospitals of the City of Havana. The ideal parasite concentration to inoculate turned out to be $8 \times 10^{6}$. The liver was the most affected organ, with a significant difference $(\mathrm{p}<0,01)$ from the rest of the organs. Through Kruskal-Wallis test it was possible to observe that there were significant differences $(p<0,0001)$ among the score obtained by each mice group, depending on the kind of isolation, according to the clinical classification of origin. This demonstrated a possible correlation between the degree of virulence of each isolate and the clinical characteristics of the disease-carrying patients.

Key words: Trichomonas vaginalis; Adolescents; Virulence.

Palabras claves: Trichomonas vaginalis, Adolescentes, Virulencia.

\section{Introducción}

Trichomonas vaginalis, es el agente causal de la forma más común de enfermedad parasitaria, sexualmente transmitida, que afecta a hombres y mujeres; cada año alrededor de 170 millones de mujeres son afectadas por este protozoo en el mundo ${ }^{1}$. Este parásito puede ser la causa de vaginitis, cervicitis y uretritis en mujeres, favoreciendo el desarrollo de una neoplasia cervical, y prostatitis, uretritis no gonocóccica y otros síndromes del tracto genitourinario bajo en hombres $^{2-4}$. En ocasiones es asintomática en ambos sexos, aunque esto sucede con mayor frecuencia en los hombres. Su control es importante, teniendo en cuenta la elevada incidencia de infecciones agudas, complicaciones y secuelas, así como su impacto socioeconómico y el papel que pueda jugar como cofactor en la transmisión del virus de inmunodeficiencia humana ${ }^{5,6}$.

La gran variabilidad en su forma de presentación y/o en la evolución de la tricomoniasis, así como la disímil respuesta de algunos pacientes al tratamiento con metronidazol como antimicrobiano de elección, condujo a la hipótesis de que existen diferentes grados de virulencia en distintos aislamientos de $T$. vaginalis, teniendo en consideración que la severidad de la infección depende además del estado inmunológico del individuo. Las investigaciones en este sentido se han llevado a cabo mediante la inoculación subcutánea del parásito en ratones, inoculación que se traduce en un absceso subcutáneo de magnitud y volumen dependientes de la virulencia de la 
cepa inoculada ${ }^{7}$. La inoculación por vía intraperitoneal ${ }^{8-10}$ también ha sido practicada; esta vía aporta mayor información sobre las alteraciones patológicas en el animal de experimentación. En 1997 Nogal y colaboradores propusieron la cepa NMRI de ratón como un buen modelo biológico con estos fines ${ }^{9}$. La inoculación intraperitoneal en ratones causa peritonitis fibropurulenta, con abscesos y focos de necrosis en los órganos abdominales y producción de líquido ascítico ${ }^{8,10}$. Para la evaluación y cuantificación de los daños ocasionados en el animal de experimentación se han empleado el índice de virulencia de Cavier ${ }^{11}$ y los criterios de Toyos ${ }^{12}$. Nuestro objetivo fue correlacionar la virulencia de diferentes cepas de T. vaginalis en el modelo animal, con la expresión clínica de la infección en un grupo de adolescentes femeninas. Para ello se ensayó la inoculación intraperitoneal de T. vaginalis en ratones NMRI de 40 aislamientos de $T$. vaginalis procedentes de un grupo de adolescentes femeninas que clínicamente fueron clasificadas en asintomáticas, sintomáticas leves, moderadas o severas.

\section{Materiales y Métodos}

Universo de trabajo. Fue estudiado un total de 575 adolescentes, atendidas en las consultas Infanto-Juvenil, de Interrupción de Embarazo y de Infecciones de Transmisión Sexual (ITS), de los Hospitales Gineco-obstétricos "Ramón González Coro" y "Eusebio Hernández" de Ciudad de La Habana, de las cuales 76 (13,2\%) tenían cultivo positivo para $T$. vaginalis. Después de un riguroso interrogatorio donde se incluyeron entre otras, preguntas relacionadas con la sintomatología clínica que pudiera guardar relación con la parasitosis, se realizó un exhaustivo examen ginecológico y, de acuerdo a la severidad de la presentación clínica, se clasificó a las pacientes en cuatro categorías, en base a criterios preestablecidos por G. Garber y cols ${ }^{13}$ modificados ${ }^{14}$, como se explica a continuación:

Se tuvo en cuenta un total de 9 parámetros donde se incluyeron signos y síntomas, asignándose un punto por la presencia de cada una de ellos: prurito, presencia de flujo vaginal, color del flujo vaginal, dolor en bajo vientre, disuria, eritema vulvar, eritema vaginal, eritema cervical y dispareunia. Una puntuación adicional de 0 a 3 puntos fue otorgada según la severidad de cada uno de los parámetros (leve, moderada o severa) con 1, 2 ó 3 puntos respectivamente, lo que permitió al final hacer la siguiente clasificación:
0: asintomática; 1 - 9: infección leve; 10 - 18: infección moderada; 19 - 27: infección severa.

Aislamiento y axenización de los parásitos. A partir de las muestras de exudado vaginal de las pacientes que resultaron ser positivas se logró axenizar un total de 52 aislamientos del parásito (12 asintomáticas y 40 sintómaticas divididas en 11 sintomáticas leves, 13 sintomáticas moderadas y 16 sintomáticas severas) por traspasos sucesivos diarios en cultivo in vitro, utilizando el medio de cultivo de Diamond TYI-S-33 ${ }^{15}$ suplementado con suero de ternera, descomplementado y sin calostro, al cual se le añadió penicilina $G$ sódica $100 \mathrm{UI} / \mathrm{ml}$ estreptomicina $30 \mu \mathrm{g} / \mathrm{ml}$ y nistatina $60 \mu \mathrm{g} / \mathrm{mL}$ de medio), hasta lograr su completa axenización. Luego fueron almacenados en nitrógeno líquido a temperaturas de $-190^{\circ}$ $\mathrm{C}$ hasta su posterior utilización. Para este estudio se empleó un total de 40 aislamientos de $T$. vaginalis (10 aislamientos por cada grupo clínico), seleccionados al azar.

Animales de experimentación. Para la realización de los experimentos se empleó ratones NMRI machos, de 6 semanas de nacidos, con un peso aproximado entre 20 y 22 gramos, mantenidos bajo los requerimientos para la especie, en condiciones ambientales controladas (temperatura y humedad), además de proporcionarles agua y alimentación adecuada.

Controles. En cada experimento se empleó como control 5 ratones no inoculados y 5 inoculados con medio sin suero, que fueron sacrificados y examinados al final de cada experimento (10 animales en cada grupo de aislamientos).

Con el objetivo de corroborar si el daño provocado al animal era producido por la acción del parásito vivo se inoculó por vía intraperitoneal dos tipos de suspensiones parasitarias: un grupo de 10 ratones se inoculó con parásitos muertos pero íntegros, cuya muerte fue provocada por un ciclo de congelación-descongelación y otros 10 ratones fueron inocularon también con parásitos muertos pero lisados, para lo cual fueron sometido a 15 ciclos también de congelación-descongelación.

Determinación del inóculo. Para determinar la concentración parasitaria ideal para su inoculación intraperitoneal, fueron evaluados tres concentraciones parasitarias del orden de: $4 \times 10^{6}$, $8 \times 10^{6}$ y $1 \times 10^{7}$ parásitos $/ \mathrm{mL}$. Se inoculó un total de 10 ratones por cada concentración parasitaria, evaluando como único parámetro la mortalidad.

Inoculaciones y análisis posterior. Una vez determinada la concentración ideal, por cada aislamiento se inoculó diez ratones (400 en total) 
utilizando la vía intraperitoneal. De acuerdo a experiencias de otros investigadore ${ }^{10}$ se decidió prefijar el séptimo día como el momento ideal para el sacrificio, el cual fue realizado mediante la administración de una sobredosis de anestesia. Una vez sacrificados se procedió al examen macroscópico de las cavidades torácica, abdominal y pelviana en busca de lesiones, las cuales fueron corroboradas macro y microscópicamente en el Departamento de Anatomía Patológica del Instituto de Medicina Tropical "Pedro Kourí'. Los animales que murieron y no pudieron ser examinados por su avanzado estado de descomposición se eliminaron de la investigación.

Diseño estadístico. El índice de virulencia de los aislamientos fue determinado al séptimo día post-inoculación, de acuerdo a los criterios empleados por Toyos ${ }^{12}$ con algunas modificaciones. Para esto se confeccionó una base de datos en la cual se anotó la puntuación correspondiente a cada parámetro, de acuerdo a las lesiones provocadas en cada uno de los órganos de los ratones examinados, según se muestra en la Tabla 1.

Según esta clasificación, la cuantificación del daño producido por cada aislamiento en un solo ratón (índice de virulencia) puede alcanzar una puntuación máxima de 42 puntos.

Los resultados fueron procesados con ayuda del paquete estadístico para Windows (GraphPad Prism versión 3.00 para Windows, GraphPad Software, San Diego California, 1999). Se utilizó la prueba no paramétrica de Kruskal-Wallis para comparar las medias de las puntuaciones obtenidas por los aislamientos de cada grupo clínico en cada parámetro analizado individualmente (ascitis, hígado, bazo, riñones, estómago, páncreas, in- testino, peritoneo, cara visceral del diafragma, cara parietal del diafragma) y para comparar las puntuaciones totales obtenidas por los aislamientos de cada grupo clínico. Se utilizó además una prueba de comparación de proporciones (EPINFO, versión 6.04a, 1996, CDC Atlanta, Georgia, USA; OMS, Ginebra, Suiza) para comparar la frecuencia de aparición de lesiones en los órganos más frecuentemente afectados. Se consideraron significativos los valores de p menores que 0,01.

\section{Resultados}

Al analizar la cavidad abdominal, torácica y pelviana de los ratones inoculados como grupos controles, no se encontró alteraciones o daños en sus órganos, relacionados con las diferentes preparaciones inoculadas como controles. De la misma forma, tampoco se encontró algún tipo de lesión en el grupo de ratones no inoculados.

Se pudo establecer que la concentración ideal para lograr que el mayor número de animales llegara vivo y con lesiones cuantificables al día de sacrificio (el séptimo día) fue la correspondiente a $8 \times 10^{6}$ parásitos $/ \mathrm{mL}$ pues, a mayor concentración $\left(1 \times 10^{7}\right.$ parásitos $\left./ \mathrm{mL}\right)$, todos los ratones fallecieron en el plazo de 4 a 7 días (Figura 1) mientras con la concentración inferior $\left(4 \times 10^{6}\right.$ parásitos $/ \mathrm{mL}$ ) no se pudo observar diferencias entre los aislamientos, en el tiempo establecido para su observación.

De un total de 400 ratones inicialmente inoculados con concentración parasitaria de $8 \times 10^{6}$ parásitos/mL, 12 murieron (2, 4 y 6 de los grupos de sintomáticos leves, moderados y severos,

Tabla 1. Criterios de puntuación empleados para evaluar las alteraciones y daños encontrados en cada ratón

\begin{tabular}{lcc}
\hline Parámetro & Intensidad del daño & Puntuación asignada \\
\hline Líquido ascítico & $<1 \mathrm{~mL}$ & 2 puntos \\
& $1-2 \mathrm{~mL}$ puntos \\
& $>2 \mathrm{~mL}$ puntos \\
• Hígado & & $0-2-4$ puntos \\
• Bazo & & $0-2-4$ puntos \\
• Riñón & & $0-2-4$ puntos \\
• Estómago & & $0-2-4$ puntos \\
• Páncreas & & $0-2-4$ puntos \\
• Intestino & & $0-2-4$ puntos \\
• Peritoneo & & $0-2-4$ puntos \\
• Cara visceral del diafragma & & $0-2-4$ puntos \\
• Cara parietal del diafragma & & $0-2-4$ puntos \\
• Lesiones en las vísceras & Ausencia de lesión & 0 puntos \\
& Abscesos & 2 puntos \\
\hline
\end{tabular}


respectivamente) y no pudieron ser analizados por su estado de descomposición, de modo que se analizó un total de 388 ratones. Al realizar la disección, se observó una gama de lesiones que fueron desde un absceso en la superficie del órgano hasta la destrucción total del parénquima.

En la Figura 2 se muestran las medias aritméticas de los valores obtenidos por las lesiones ocasionadas en los ratones, de acuerdo al tipo de aislamiento. En todos los parámetros analizados pudo comprobarse la existencia de diferencias estadísticamente significativas $(p<0,0001)$ en el índice de virulencia provocado en el animal experimental, según tipo de aislamiento inoculado. En el grupo de ratones inoculados con aislamientos de pacientes asintomáticos la manifestación patológica más frecuente fue la presencia de líquido ascítico.

En la Tabla 2 se reflejan la frecuencia de aparición de daños más frecuentes encontrados en cada uno de los órganos al examinar la cavidad abdominal, torácica y pelviana de cada ratón, teniendo en cuenta los criterios de puntuación empleados para evaluar las alteraciones y daños en cada animal, según el grupo clínico de procedencia del aislamiento inoculado. El hígado fue el órgano más frecuentemente afectado, y se diferenció significativamente $(\mathrm{p}<0,01)$ del resto de los órganos.

$\mathrm{Al}$ analizar la media geométrica y desviación
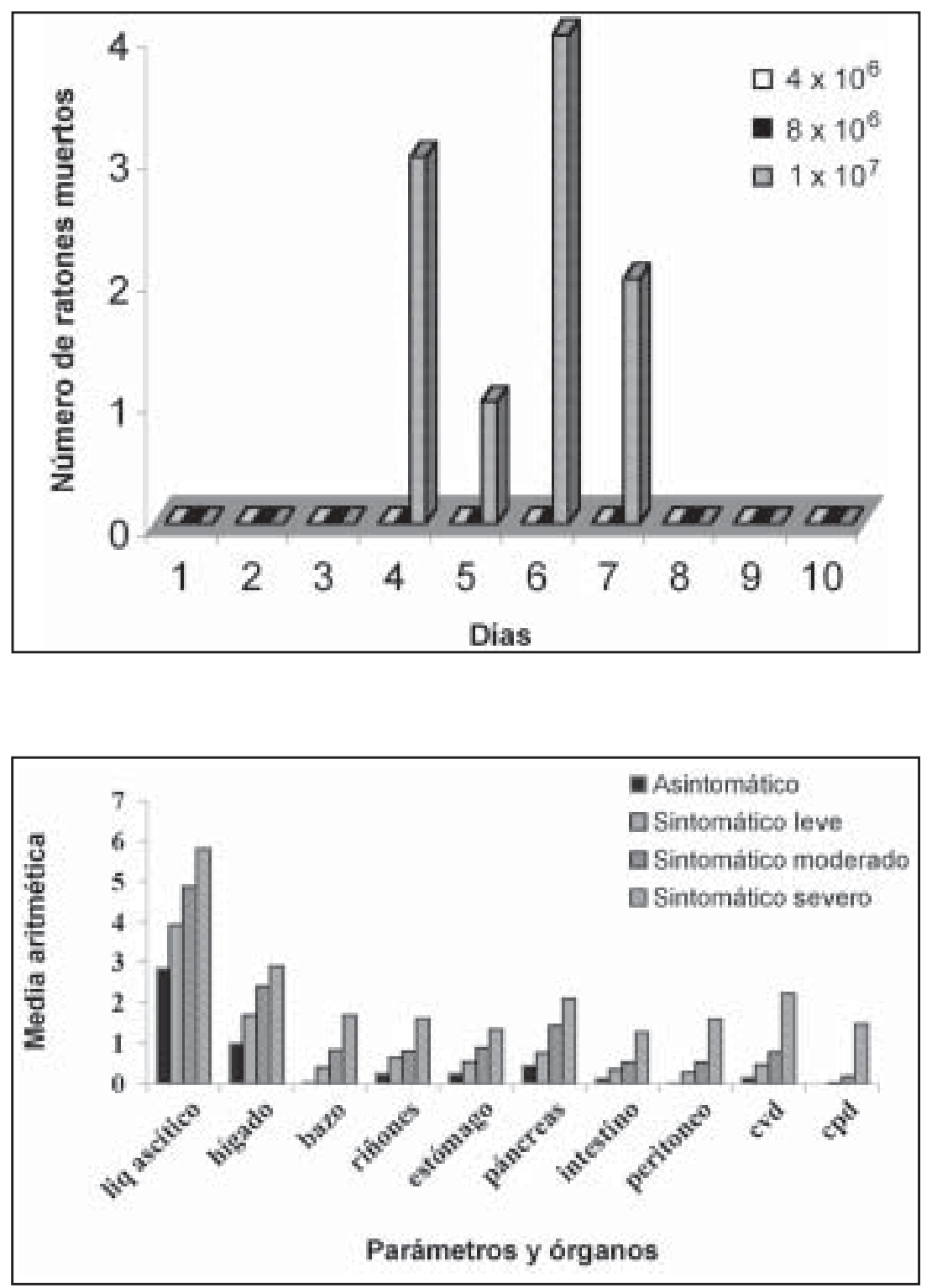

Figura 1. Efecto de la utilización de diferentes concentraciones de $T$. vaginalis sobre la mortalidad en ratones inoculados por vía intraperitoneal.
Figura 2. Media aritmética de los valores obtenidos por las lesiones ocasionadas en los ratones de acuerdo al tipo de aislamiento ( $\mathrm{p}<0,0001)$. cvd: cara visceral del diafragma cpd: cara parietal del diafragma. 
Tabla 2. Frecuencia de aparición de lesiones abdominales en ratones inoculados por vía intraperitoneal con $T$. vaginalis, expresada en porcentaje, según categoría clínica de los aislamientos

\begin{tabular}{lcccc}
\hline Órgano & $\begin{array}{c}\text { Asintomática } \\
(\mathbf{n = 1 0 0})\end{array}$ & $\begin{array}{c}\text { Intensidad de la tricomoniasis vaginal* } \\
\text { Leve } \\
(\mathbf{n = 9 8})\end{array}$ & $\begin{array}{c}\text { Moderada } \\
(\mathbf{n = 9 6 )}\end{array}$ & $\begin{array}{c}\text { Severa } \\
(\mathbf{n = 9 4 )}\end{array}$ \\
\hline Hígado & 40 & 65,30 & 82,29 & 100 \\
Bazo & 3 & 20,40 & 40,62 & 79,78 \\
Riñón & 13 & 32,65 & 39,58 & 79,78 \\
Estómago & 11 & 22,44 & 42,7 & 64,89 \\
Páncreas & 18 & 36,73 & 61,45 & 74,46 \\
Intestino & 7 & 17,34 & 25,0 & 61,7 \\
\hline
\end{tabular}

* Definiciones en Materiales y Método

$\mathrm{p}<0,01$ (Prueba de Kruskal Wallis)

Tabla 3. Media aritmética y desviación estándar de la puntuación obtenida en el modelo experimental de acuerdo a la categoría clínica de los aislamientos

\begin{tabular}{lccc}
\hline Clasificación de los aislamientos & $\begin{array}{c}\text { Puntuación obtenida en los modelos experimentales } \dagger \\
\mathbf{N}^{\circ} \text { de ratones }\end{array}$ & $\overline{\mathbf{x}}$ & $( \pm$ DS $)$ \\
\hline Asintomático & 100 & 5,2 & $(3,54)$ \\
Sintomático leve & 98 & 9,2 & $(4,54)$ \\
Sintomático moderado & 96 & 13,3 & $(5,90)$ \\
Sintomático severo & 94 & $22,1 \quad(6,36)$ \\
\hline
\end{tabular}

$\uparrow$ Se inocularon hasta 10 ratones por cada aislamiento.

$\mathrm{p}<0,0001$ (Prueba de Kruskal-Wallis).

estándar de la puntuación obtenida en los modelos experimentales de acuerdo a la categoría clínica de los aislamientos, se pudo observar mediante la prueba de Kruskal-Wallis que existen diferencias significativas $(\mathrm{p}<0,0001)$ entre las puntuaciones alcanzadas por cada grupo, en dependencia del tipo de aislamiento (Tabla 3).

\section{Discusión}

Con la finalidad de medir la virulencia in vivo de $T$. vaginalis se ha ensayado la infección experimental mediante inoculación extravaginal del parásito por vías subcutánea e intraperitoneal; cuál de las dos resulta más útil es controversial. En nuestro estudio escogimos la vía intraperitoneal teniendo en consideración que aporta un mayor grado de información relacionada con las lesiones de órganos y cavidades adyacentes, tal y como ha sido sugerido por otros investigadores ${ }^{11,12}$ en lugar de una deposición pasiva en la piel cuando se emplea la vía subcutánea, que sólo aporta información muy localizada.
El hecho de no haber encontrado alteraciones en los grupos controles habla a favor de que las alteraciones y daños encontrados en la cavidad abdominal de los ratones inoculados con los parásitos vivos fueron resultado del metabolismo activo del parásito que fue capaz de colonizar y sobrevivir en la cavidad, lo que fue confirmado por la observación microscópica del parásito móvil en las lesiones. La inyección de parásitos utilizando medio carente de suero tuvo por objeto facilitar la acción de las proteasas que desenmascaran las adhesinas localizadas sobre la superficie del parásito, las cuales le permiten unirse a las células del huésped ${ }^{16}$.

La concentración parasitaria ideal fue del orden de $8 \times 10^{6}$ parásitos $/ \mathrm{ml}$ con lo que logramos que el mayor número de animales llegara vivo al día del sacrificio. Cavier y cols ${ }^{11}$ emplearon inóculos de $1 \times 10^{6}$ en sus estudios de virulencia en los que contemplaron la mortalidad de los animales como un importante factor relacionado con la virulencia, con el inconveniente de tener que extender sus ensayos hasta tres semanas de observación. Otros autores como Gold ${ }^{8}$ utiliza- 
ron inóculos con un número de parásitos del orden de 1 x $10^{7}$, y también sacrificaron los animales al séptimo día. En nuestra experiencia esta concentración parasitaria por inoculo no resultó útil por la cantidad de animales muertos antes del día designado para el sacrificio.

Aunque no hubo diferencia estadísticamente significativa $(p>0,17)$ es necesario precisar que los ratones muertos en el curso del ensayo pertenecían al grupo inoculado con los aislamientos procedentes de pacientes sintomáticas; cabe plantear la posibilidad de que esto guarde relación con una mayor virulencia de las cepas, más aún al considerar que la mayor mortalidad se produjo entre los ratones inoculados con cepas provenientes del grupo de pacientes sintomáticas severas (datos no mostrados).

$\mathrm{Kulda}^{17}$, en sus estudios donde sólo mide mortalidad, plantea que la gran mayoría (80-95\%) de los aislamientos de $T$. vaginalis seleccionados al azar pueden producir la muerte de un ratón después de la inoculación intraperitoneal y que esta propiedad debe ser tenida en cuenta en los ensayos realizados en el laboratorio. El propio autor plantea que el número de T. vaginalis inoculadas para que necesariamente causen infección letal puede ser diferente, en dependencia del tipo de cepa. El curso de la infección puede variar con la virulencia de la cepa parasitaria empleada y con la susceptibilidad del modelo animal. Algunos animales pueden morir antes que la lesión esté completamente desarrollada; en otras oportunidades la infección no es fatal, las lesiones están limitadas, no se extienden demasiado ni aparecen en varios órganos al mimo tiempo, en dependencia del tipo de cepa inoculada.

Se describe que los cambios histopatológicos que acompañan a una infección intraperitoneal en ratones con $T$. vaginalis pueden ser básicamente divididos en tres tipos, reflejando la virulencia de la cepa utilizada. En los ratones inoculados con cepas altamente virulentas, predominan los cambios inflamatorios exudativos, en la mayoría de los casos la invasión de órganos abdominales se expresa en necrosis y la delimitación de las lesiones es muy pobre, haciéndose extensiva e invadiendo zonas en contacto con los tejidos del huésped. Los cambios patológicos provocados por cepas de moderada virulencia están asociados con una producción de exudado fibrinopurulento, los órganos abdominales son invadidos, pero la extensión de las lesiones está limitada; la peritonitis puede ser la causa de muerte. Las infecciones con cepas de baja virulencia son caracterizadas por una exudación mínima, puede existir invasión a órganos pero cuando aparece es mínima. Se dice que en este tipo de infección los parásitos pueden persistir encapsulados en la lesión por algunas semanas.

En nuestros resultados encontramos situaciones muy parecidas a las planteadas con anterioridad, indicando que existió correlación entre los índices de virulencia producidos por los aislamientos y su clasificación clínica: los ratones inoculados con aislamientos procedentes de pacientes portadores de una infección sintomática severa fueron los que más puntuación alcanzaron. Esta positiva correlación también ha sido previamente encontrada por otros investigadores ${ }^{17,18}$. El hígado fue el órgano más frecuentemente afectado pero otras vísceras también estuvieron afectadas, esto fue común para los ratones inoculados con los aislamientos de pacientes sintomáticos.

La gran variabilidad en la presentación y evolución de la tricomoniasis, así como en la respuesta al tratamiento en este grupo de adolescentes justificaron la planificación de este ensayo experimental que nos ayudó a determinar el posible grado de virulencia entre los diferentes grupos de acuerdo a la sintomatología y clasificación clínica de las pacientes de las cuales fueron aislados los parásitos. La intensidad de las lesiones provocadas en el animal parece estar relacionada con la inherente virulencia de cada aislamiento.

Aparte de nuestro interés científico en saber acerca del grado de patogenicidad in vivo de aislamientos de $T$. vaginalis, éste es el primer reporte en Cuba de un estudio que se realizó utilizando aislamientos parasitarios procedentes de un grupo de 40 adolescentes, estudio que nos ha permitido a la vez acercarnos al comportamiento clínico de esta parasitosis en nuestro medio, en un grupo humano en que la tricomoniasis tiene alta trascendencia por su transmisión sexual y porque puede acarrear serias consecuencias, de no ser atendida a tiempo.

\section{Resumen}

Se estudió la correlación entre intensidad clínica de la tricomoniasis vaginal en adolescentes y la virulencia de Trichomonas vaginalis en un modelo experimental. Para ello fueron inoculados por vía intraperitoneal en ratones NMRI, 40 aislamientos de T. vaginalis obtenidas a partir de exudados vaginales provenientes de adolescentes, clínicamente clasificadas como asintomáticas, y sintomáticas leves, moderadas o severas, atendidas en las consultas Infanto-Juvenil, de Interrupción de Embarazo y de Infecciones de Transmisión Sexual (ITS), de hospi- 
tales Gineco-obstétricos de Ciudad de La Habana. La concentración parasitaria ideal a inocular fue del orden de $8 \times 10^{6}$. El hígado fue el órgano más afectado y se diferenció significativamente $(\mathrm{p}<0,01)$ del resto de los órganos. Se pudo observar mediante la prueba de Kruskal -Wallis que existieron diferencias significativas $(\mathrm{p}<0,0001)$ entre la puntuación obtenida por cada grupo de ratones según el tipo de aislamiento de acuerdo a la clasificación clínica de procedencia, lo que demostró una posible correlación entre el grado de virulencia de cada aislamiento y las intensidad clínica de tricomoniasis vaginal en adolescentes.

\section{Bibliografía}

1.- World Health Organization. An overview of selected curable sexually transmitted diseases, p 2-27. In World Health Organization (ed.), In Global Program on AIDS.1997 World Health Organization, Geneva, Switzerland.

2.- Zhang Z F, Begg C B. Is T. vaginalis a cause of cervical neoplasia: result from a combined analysis of 24 studies. Int J Epidemiol 1994; 23: 682-90.

3.- Gram I T, Macaluso M, Krurchil J, Stalberg H. Trichomonas vaginalis and Human papillomavirus infection and the incidence of cervical intraepithelial neoplasia grade III. Cancer Causes and Control 1992; 3: 231-6.

4.- Viikki M, Pukkala E, Nieminen P, Hakama M. Gynaecological infections as risk determinants of subsequent cervical neoplasia. Acta Oncol 2000; 39: 71-5.

5.- Sorvillo F, Kerndt P. Trichomonas vaginalis and amplification of HIV-1 transmission. Lancet 1998; 351: $213-4$

6.- Laga M, Manoka A, Kivuvu M, Malele B, Tuliza M, Nzila N, et al. Non ulcerative sexually transmitted diseases (STD) as risk factors for HIV-1 transmission in women: result of cohort study. AIDS, 1993; 7: 95-102.

7.- Honigberg B M. Comparative pathogenicity of $T$. vaginalis and T. gallinae to mice, a gross pathology, quantitative evaluation of virulence, and some factors affecting pathogenicity. J Parasitol 1961; 47: 545-71.

8.- Gold T. Trichomonas vaginalis: strain differences in adhesion to plastic and virulence in vitro and in vivo. Springer Verlag. Parasitol Res 1993; 79: 309-15.

9.- Nogal Ruiz J J, Escario J A, Martínez Díaz R A, Gómez Barrio A. Evaluation of murine model of experimental trichomoniasis. Parasite 1997; 2: 127-32.

10.- Escario J A, Gómez Barrio A, Martínez Fernández A $\mathrm{R}$. The relationship of experimental pathogenicity in vivo with in vitro cytoadherence and cytotoxicity of 6 different isolates of $T$. vaginalis. Intern J Parasitol 1995; 25: 999-1000.

11.- Cavier R E, Gobert J G, Savel J. Application d'une methode d'infestation intraperitoneale de la souris par T. vaginalis a l'etude pharmacologique des trichomonacides. Annales Pharmaceutiques Francaises 1972; 30: 637-42.

12.- Toyos J M. Patogenia experimental de T. vaginalis Donné 1836 en el ratón Mus musculus. Anales de la Facultad de Veterinaria de León 1974; 20: 35-90.

13.- Garber G, Lemchuk-Favel L. Association of production of cell detaching factor with the clinical presentation of Trichomonas vaginalis. J. Clin Microbiol 1990; 28: $2415-7$

14.- Rojas Rivero L, Izquierdo A, Sarría C, Sariego I, Fraga J. Comportamiento de la trichomonosis vaginal en un grupo de adolescentes. Rev Cub Med Trop 2003; 55: $69-74$

15.- Diamond L S, Harlow D R, Cunich C C. A new medium for the axenic cultivation of Entamoeba histolytica and other Entamoeba. Trans R Soc Trop Med Hyg 1978; 72: 431-2.

16.- Arroyo R, Alderete J F. Trichomona vaginalis surface proteinase activity is necessary for parasite adhererence to epithelial cells. Infect Immun 1989; 57: 2991-7.

17.- Kulda J. Employment of experimental animals in studies of $T$. vaginalis infection. In: Trichomonads parasitic in humans. Honigberg B M, Springer-Verlag, New York, 1990: 112-54.

18.- Vanacova S, Tachezy J, Kulda J, Flegr J. Characterizacion of trichomonad species and strains by fingerprinting. J Euk Microbiol 1997; 44: 545-52.

Correspondencia a:

Lázara Rojas Rivero

E-mail: 1rojas@ipk.sld.cu 